\title{
Genomic structure and chromosomal mapping of the mouse transcription factor TEF-5 (Tead3) gene
}

\author{
Patrick Jacquemin, ${ }^{1,2 *}$ Zhi Chen, ${ }^{3}$ Joseph A. Martial, ${ }^{2}$ Irwin Davidson ${ }^{1}$ \\ ${ }^{1}$ Institut de Génétique et de Biologie Moléculaire et Cellulaire, CNRS/INSERM/ULP, 1, rue Laurent Fries, F-67404 Illkirch Cédex, France \\ ${ }^{2}$ Laboratoire de Biologie Moléculaire et de Génie Génétique, Institut de Chimie, Université de Liège, B-4000 Sart-Tilman, Belgique \\ ${ }^{3}$ Cardiovascular Research Center, University of Michigan, Ann Arbor, Michigan 48109-0644, USA
}

Received: 21 September 1998 / Accepted: 31 January 1999

Four members of the Transcriptional Enhancer Factor (TEF) family of transcription factors have been identified in human and mouse (TEF-1, TEF-3, TEF-4, and TEF-5; approved gene symbols TEAD1, TEAD4, TEAD2, and TEAD3 respectively; Yasunami et al. 1995, 1996; Jacquemin et al. 1996, 1997; Yockey et al. 1996; Hsu et al. 1996; Kaneko et al. 1997; reviewed in Jacquemin and Davidson 1997). These factors possess the TEA DNA binding domain which recognizes degenerate sites in the enhancers and promoters of several viral and cellular genes. The TEFs comprise a short variable $\mathrm{N}$-terminal region preceding the highly conserved TEA domain, a variable hydrophobic region immediately after the TEA domain, and a large, well-conserved C-terminal domain (see Fig. 1A).

Expression of the murine (m)Tead factors is differentially regulated during development, the principal sites of expression being mitotic neuroblasts, skeletal and cardiac muscle, the placenta, lung, and several other viscera (see Jacquemin and Davidson 1997). Insertional mutagenesis showed that Teadl is essential for cardiac development (Chen et al. 1994). Tead3 is first expressed in extraembryonic tissues such as the giant trophoblastic cells, and expression persists in the giant cells and labyrinthine region of the placenta throughout gestation (Jacquemin et al. 1998). Tead3 expression is limited to the extraembryonic layers until around 9.5 days post coitum, when further expression in epithelia and developing viscera is also observed.

As gene targeting experiments will be required to further define the role of Tead 3 in placental development, we report here as a first step the isolation and characterization of the Tead3 gene and the determination of its chromosomal location.

A $\lambda$ EMBL3 mouse genomic DNA library (kindly provided by J.-M. Garnier) was screened with a mix of the cDNAs encoding the Tead1, $-2,-3$ and -4 TEA domains as probes. After isolation, phages containing Tead3 were identified by PCR using Tead3specific primers, and their DNA was analyzed by Southern blotting with the full-length Tead 3 cDNA. Following digestion with $P v u I I$, 4 hybridizing fragments ranging from 1.1 to $3.2 \mathrm{~kb}$ were cloned. These fragments were entirely sequenced and comparison with the Tead 3 cDNA allowed identification of the introns and exons. Junctions between the fragments, and sequences upstream and downstream of the coding region were obtained by direct sequencing on the phage DNA. A total sequence of 12,622 base pairs (bp) was obtained.

*Present address: Unité HORM, UCL-ICP, 75 Av. Hippocrate, B-1200 Bruxelles, Belgique.

The nucleotide sequence described in this manuscript has been assigned the accession number AJ131526 in the EMBL database.

Correspondence to: I. Davidson
Analysis of this sequence indicates that the Tead3 gene comprises 13 exons (the smallest is $12 \mathrm{bp}$ ) and 12 introns (from $91 \mathrm{bp}$ to 3582 bp; Fig. 1A). All of the intron-exon junctions conform with the GT/AG rule. The TEA DBD is encoded by three exons (II-IVA), each coding for one of the three putative $\alpha$-helices. In chicken (c) two Tead3 isoforms have been characterized with differences in the C-terminal $\alpha$-helix of the TEA DBD encoded by exon IV. These isoforms may be generated by differential use of a duplication of this exon. Indeed, downstream of exon IVA, we found a variant copy of this exon (IVB, Fig. 1B). So far only exon IVA has been found in mammalian Tead 3 cDNAs. In contrast, in TEAD1, only exon IVB has been found (Fig. 1B). However, in one exceptional case a TEAD1 cDNA from HeLa cells that we characterized previously contained a duplicated region at the end of the TEA domain corresponding to a splice variant in which both exons IVA and IVB were present (our unpublished data and see Fig. 1B). This TEAD1 variant was still able to specifically bind DNA in electrophoretic mobility shift assays (our unpublished data).

Alternative splicing of the four amino acids encoded by exon $\mathrm{V}$ was also observed. This exon was present in all the isolated mouse or human TEAD3 cDNAs, but was variable in TEAD1.

Comparison of the Tead3 locus with that of Tead2 (Suzuki et al. 1996) showed that the overall organization of the genes is conserved. The location of the intron-exon junctions for Tead3 relative to the peptide sequence is shown in Fig. 2A. All the junctions are in analogous position to those of Tead2 with the exception of the upstream and downstream borders of exon VII. A comparison of the exon VII frontiers for Tead 2 and -3 is shown in Fig. 2B. This exon encodes part of the most variable region of the TEAD family.

We further determined the chromosomal localization of Tead3, using an interspecific backcross DNA panel (The Jackson Laboratory; Bar Harbor, Me; Rowe et al. 1994). The panel was generated by using DNA from 94 backcross animals from the reciprocal cross $(\mathrm{C} 57 \mathrm{BL} / 6 \mathrm{JEi} \times \mathrm{SPRET} / \mathrm{Ei}) \mathrm{F}_{1} \times \mathrm{SPRET} / \mathrm{Ei}$ ("Jackson BSS" backcross panel). Southern blot analysis with a Tead 3 cDNA probe detected a restriction fragment length variation (RFLV) between DNAs of the C57BL/6J and $M$. spretus. The probe detected a 2-kb BamHI fragment in C57BL/6 DNA and a 5-kb fragment in $M$. spretus DNA (data not shown). Therefore, the RFLV identified was used to follow the segregation of the Tead3 locus in the Jackson BSS backcross panel.

The resulting data show that Tead3 is 1.1 centimorgans (cM) distal to the D17WSU92e locus, $2.1 \mathrm{cM}$ and $3.2 \mathrm{cM}$ proximal to the Tff3 (intestinal trefoil factor 3) or D17Mit16 locus respectively, near the proximal end of Chr 17 (Fig. 3). The Fkbp5 gene (FK506 binding protein 5) is also present in this region of Chr 17. In fact, Tead 3 co-segregates with Fkbp5 gene in all 94 animals. Thus, we 
A

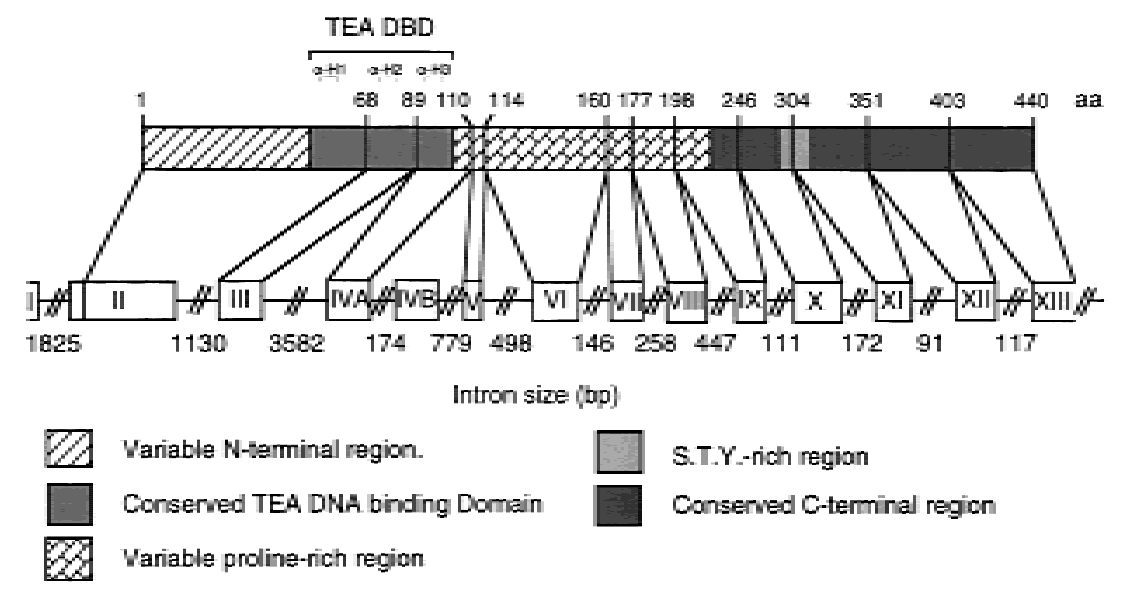

B

Intron/Exon Organisation of Tead3.

TEA DBD
Fig. 1. (A) Genomic structure of Tead3. Complete exon-intron organization of Tead 3 gene relative to the amino acid (aa) sequence is indicated. The three putative $\alpha$-helices of the TEA DNA-binding domain are shown. (B) Comparisons of the sequences of the alternative exons encoding the putative $\alpha$-helix 3 of the TEA domains of chicken (c) and mouse (m) Tead 3 and human TEAD1.
A

IASNSWTANS SPGEAREDGS EGLDKGLDND AEGVWSPDIE QSFQEALAIY

III

IVA

PPCGRRKIIL SDEGKMYGRN ELIARYIKLR TGKTRTRKO্বV SSHIQVLARK $\mathrm{V}$

VI

KVREYQVGIKT AMNTTQVSKD KALQSMASMS SAQIVSASVI QNKFSPPSPL VII

VIII

PQAVFSSSSRTFWSPPLVGQ QPGPSQDIKP FAQPAYPIQP PLPPALN\$YY

IX

SLAPLPPAAA SATASAPAWQ DRTIASSRLR LLEYSAFMEV QRDPDTYSKH

$\mathrm{X}$

LFVHIGQTNP AFSDPPLEAV DVRQIYDKFP EKKGGLKELY EKGPPNAFFL

$\mathrm{XI}$

VKFWADLNST IQEGPGAFYG VSSQYSSADS MTISVSTKVC SFGKQVVEKV

XII

FTEYARLENG RFVYRIHRSP MCEYMINFIH KLKHLPEKYM MNSVLENFTI

XIII

LQTVVTSRDS QETLLVIAFV FEVSTSEHGA QHHVYKLVKD
B

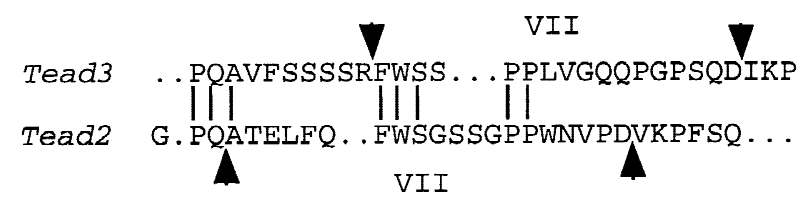

Fig. 2. (A) Location of the different exons on the Tead3 amino acid sequence. Transition between the different exons is indicated. (B) Comparison of the exon VII frontiers for Tead 3 and Tead2. Exon VII is located in the most variable region between the different Teads. 


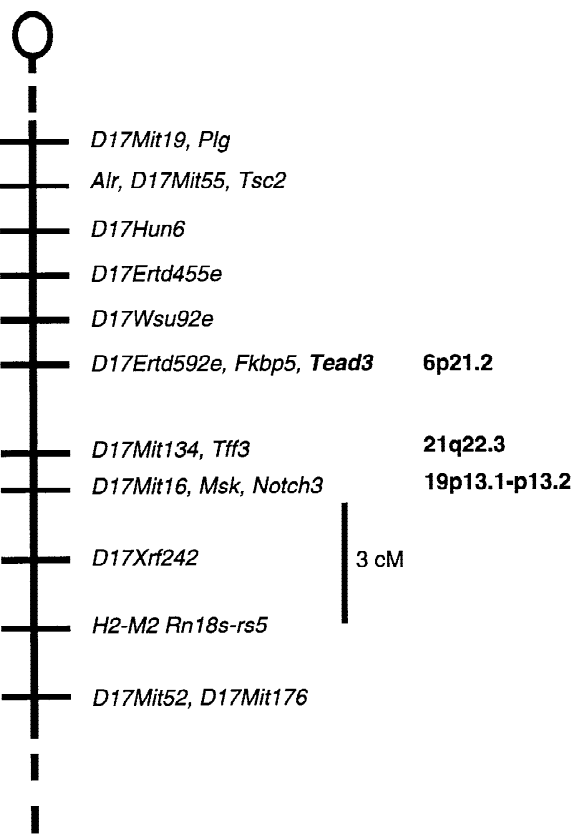

Jackson BSS Chromosome 17

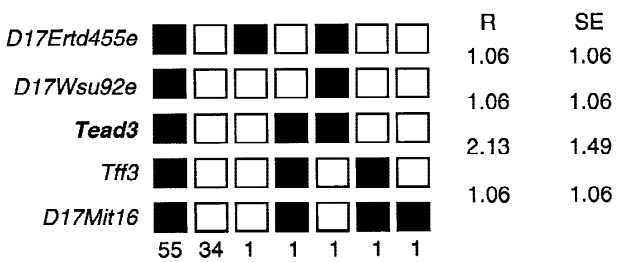

Fig. 3. The Tead 3 locus maps in the proximal region of mouse Chr 17. Tead3 was mapped to mouse $\mathrm{Chr} 17$ by interspecific backcross analysis with the Jackson BSS panel. A partial Chr 17 linkage map showing the location of Tead3 in relation to linked genes or physical markers is shown. A scale bar of $3 \mathrm{cM}$ is shown to the right of the chromosome. Where known, the chromosomal locations of human homologs of neighboring genes is indicated also on the right. The haplotype analysis, along with the number of animals used, the recombination frequency $(\mathrm{R})$, and the standard error (SE), is shown below the figure.

conclude that Tead3 is in close proximity to Fkbp5 near the proximal end of Chr 17.

The location of Tead3 is syntenic to that of TEAD3, which we previously mapped to 6p21.2 of Chr (Jacquemin et al. 1999). However, Tff3, located closed to Tead3, is located on human Chr 21. Consequently it appears that the end of syntenicity between human Chr 6 and mouse Chr 17 must lie between these two loci.

In conclusion, these results indicate that the genomic organisation of four TEAD genes is most probably very well conserved. These data could be useful for designing a common scheme for knock-out strategies of the different TEAD genes.
Acknowledgments. We thank P. Chambon for support, L. Carré for technical assistance, S. Vicaire for DNA sequencing, and the oligonucleotide facility. P. Jacquemin was supported by a fellowship from the Fondation pour la Recherche Médicale. This work was supported by grants from the Services Féderaux des Affaires Scientifiques, Techniques, et Culturelles (Belgium) PAI P4/30 and Actions de Recherche Concertées programme No. 95/00-193, the CNRS, the INSERM, the Hôpital Universitaire de Strasbourg, the Ministère de la Recherche et de la Technologie, the Association pour la Recherche contre le Cancer, and the Ligue National contre le Cancer.

\section{References}

Chen Z, Friedrich GA, Soriano P (1994) Transcriptional enhancer factor 1 disruption by a retroviral gene trap leads to heart defects and embryonic lethality in mice. Genes Dev 8, 2293-2301

Hsu DKW, Guo Y, Alberts GF, Copeland NG, Gilbert DJ et al. (1996) Identification of a murine TEF-1-related gene expressed after mitogenic stimulation of quiescent fibroblasts and during myogenic differentiation. J Biol Chem 271, 13786-13795

Jacquemin P, Davidson I (1997) The role of the TEF transcription factors in cardiogenesis and other developmental processes. Trends Cardiovasc Med 7, 192-197

Jacquemin P, Hwang JJ, Martial JA, Dolle P, Davidson I (1996) A novel family of developmentally regulated mammalian transcription factors containing the TEA/ATTS DNA binding domain. J Biol Chem 271, 21775-21785

Jacquemin P, Martial JA, Davidson I (1997) Human TEF-5 is preferentially expressed in placenta and binds to multiple functional elements of the human chorionic somatomammotropin-B gene enhancer. J Biol Chem 272, 12928-12937

Jacquemin P, Sapin V, Alsat E, Evain-Brion D, Dollé P et al. (1998) Differential expression of the TEF family of transcription factors in the murine placenta and during differentiation of primary human trophoblasts in vitro. Dev Dyn 212, 423-436

Jacquemin P, Depetris D, Mattei M-G, Martial JA, Davidson I (1999) Localization of human transcription factor TEF-4 and TEF-5 (TEAD2, TEAD3) genes to chromosomes $19 \mathrm{q} 13.3$ and $6 \mathrm{p} 21.2$ using fluorescence in situ hybridization and radiation hybrid analysis. Genomics 55, 127129

Kaneko KJ, Cullinan EB, Latham KE, De Pamphilis ML (1997) Transcription factor mTEAD-2 is selectively expressed at the beginning of zygotic gene expression in the mouse. Development 124, 63-73

Rowe LB, Nadeau JH, Turner R, Frankel WN, Letts VA et al. (1994) Maps from two interspecific backcross DNA panels available as a community genetic mapping resource. Mamm Genome 5, 253-274

Suzuki K, Yasunami M, Matsuda Y, Maeda T, Kobayashi H, et al. (1996) Structural organization and chromosomal assignment of the mouse embryonic TEA domain-containing factor (ETF) gene. Genomics 36, 263-270

Yasunami M, Suzuki K, Houtani T, Sugimoto T, Ohkubo H (1995) Molecular characterization of cDNA encoding a novel protein related to transcriptional enhancer factor-1 from neural precursor cells. J Biol Chem 270, 18649-18654

Yasunami M, Suzuki K, Ohkubo H (1996) A novel family of TEA domaincontaining transcription factors with distinct spatiotemporal expression patterns. Biochem Biophys Res Commun 228, 365-370

Yockey CE, Smith G, Izumo S, Shimizu N (1996). cDNA cloning and characterization of murine transcriptional enhancer factor-1-related protein 1, a transcription factor that binds to the M-CAT motif. J Biol Chem $271,3727-3736$ 